\title{
Studie 4: \\ Berufswahlkompetenzentwicklung \\ an weiterführenden \\ allgemeinbildenden Schulen: Eine \\ latente Transitionsanalyse
}

\subsection{Einleitung}

In Deutschland fühlen sich Schüler*innen an allgemeinbildenden Schulen auf dem Weg zum Abitur oftmals noch nicht ausreichend vorbereitet, um für ihre berufliche Zukunft konkrete Pläne zu entwickeln oder eine diesbezügliche Entscheidung zu treffen. In mehr als einem Viertel der Fälle fehlen ausreichende Informationen zu Studien- und Ausbildungsmöglichkeiten, um eine Entscheidung zu treffen, mit der sich die Heranwachsenden wohlfühlen (Schneider et al., 2017).

Mit $25 \%$ (BMBF, 2016, S. 72) respektive $29 \%$ (Heublein et al., 2017, S. XV) sind die Abbruchquoten in Ausbildung und Studium in Deutschland unverändert hoch. Ehemalige Auszubildende und Studierende nennen als Abbruchgrund u. a. falsche Erwartungen in Hinblick auf die Arbeitsabläufe und -anforderungen im Alltag (vgl. BMBF, 2016). Diese lassen sich durch mangelndes Wissen über die Arbeitswelt erklären. Berufsbezogenes Wissen sowie Wissen über berufliche Voraussetzungen gehören zu den für die Berufswahl relevanten Kompetenzen (Crites \& Savickas, 1995; Driesel-Lange et al., 2010).

Die Entwicklung berufswahlbezogener Kompetenzen spielt im Kontext der Identitätsentwicklung von Jugendlichen eine wichtige Rolle. Schließlich stellen sie wichtige Werkzeuge für die lebenslange Gestaltung der eigenen beruflichen Laufbahn dar (Dreher \& Dreher, 1985; Erikson, 1968; Savickas et al., 2009).

Diese empirische Studie wurde 2018 in ähnlicher Form veröffentlicht und kann wie folgt zitiert werden: Ohlemann, S., \& Driesel-Lange, K. (2018). Career competence development of students in German secondary schools: A latent transition analysis. In C. Nägele $\&$ B. E. Stalder (Eds.), Trends in vocational education and training research. Proceedings of the European Conference on Educational Research (ECER), Vocational Education and Training Network (VETNET) (pp. 261-271). doi:10.5281/zenodo.1319694 
Unterstützung im beruflichen Orientierungsprozess wird in Deutschland, wie ausgiebig in Abschnitt 2.2 dargestellt wurde, vor allem eingebettet in den schulischen Kontext angeboten. Da berufsorientierende Maßnahmen auch mit dem Ziel des Berufswahlkompetenzerwerbs durchgeführt werden, könnte die schulische Begleitung beruflicher Orientierung als eine der Einflussgrößen auf erfolgreiche Übergänge von der allgemeinbildenden in die berufliche oder tertiäre Bildung betrachtet werden.

Mit Blick auf die erwähnten Abbruchquoten stellt sich die Frage, ob die bestehenden berufsorientierenden Angebote in Schule gegenwärtig alle Schüler*innen gleich gut dabei unterstützen, sich auf die nachschulischen Übergänge vorzubereiten. Um dieses Problem zu untersuchen, müssen zunächst zwei Fragen beantwortet werden:

(1) Gibt es Gruppen mit jeweils homogenen Entwicklungsständen hinsichtlich der Berufswahlkompetenz?

(2) Wie sehen die kurz- bzw. mittelfristigen Entwicklungsprozesse in Bezug auf die Berufswahlkompetenz aus?

Darauf aufbauend können die beiden folgenden Fragen adressiert werden:

(3) Wirken sich berufsorientierende Maßnahmen unterschiedlich auf die Berufswahlkompetenzentwicklung verschiedener Profilgruppen aus?

(4) Wie könnte das Wissen um spezifische Bedarfe von Schüler*innen auf die schulische Unterstützung beruflicher Orientierungsprozesse übertragen werden?

\subsection{Aktuelle Befunde zu Berufswahlkompetenz und Profilen beruflicher Entwicklung}

Wie in der vorangegangenen Studie bereits dargestellt (siehe Kapitel 11), haben sich frühere Untersuchungen mit Punkt 1) befasst und differenzierbare Profile der beruflichen Entwicklung identifiziert. Dabei wurden vor allem übergreifende Niveauunterschiede, jedoch selten differierende Entwicklungsmuster sichtbar (Driesel-Lange \& Kracke, 2017; Hirschi \& Valero, 2015; Kaak et al., 2015; Perera \& Mcllveen, 2017).

Diese Querschnittsstudien eröffnen vielerlei Fragen, wie sich berufswahlbezogene Kompetenzen von Schüler*innen im zeitlichen Verlauf entwickeln. Daher 
konzentriert sich die folgende Analyse auf Punkt 2), in dem die Verläufe von und zwischen Profilen im Laufe der Zeit untersucht werden.

In Anknüpfung an das theoretische Modell von Driesel-Lange et al. (2010), welches die Entwicklung von Berufswahlkompetenz als individuellen und phasenhaften Prozess darstellt, wurde die Forschungsfrage nach der zeitlichen Entwicklung von Berufswahlkompetenz in drei Hypothesen unterteilt:

(1) Die berufliche Orientierung von Schüler*innen beginnt an unterschiedlichen Punkten und verläuft sowohl zeitlich als auch hinsichtlich des Entwicklungsgrads der Berufswahlkompetenz in unterschiedlichen Bahnen.

(2) Jugendliche wechseln in ihrem Orientierungsprozess zwischen Profilgruppen.

(3) Alle Schüler*innen erleben einen Kompetenzzuwachs.

\subsection{Methode}

\section{Stichprobe}

Die Analysen basieren auf Daten aus der Längsschnittstudie Pilotprojekt Potentialanalyse, die in Nordrhein-Westfalen durchgeführt wurde (Driesel-Lange \& Kracke, 2017, vgl. Abschnitt 8.1). Aufgrund der relativ kleinen Stichprobengrößen $\left(N_{\mathrm{t} 1}\right.$ $\left.=309, N_{\mathrm{t} 2}=337, N_{\mathrm{t} 3}=303, N_{\mathrm{t} 1-3}=220\right)$ und der Komplexität der Analysemethode wurden nur die ersten drei der insgesamt vier Messzeitpunkte in diese längsschnittliche Untersuchung mit einbezogen. Zum ersten Messzeitpunkt besuchten die Schüler*innen die Jahrgangsstufe neun an einem Gymnasium (52 \%) bzw. einer Gesamtschule (48\%). Zu diesem Zeitpunkt waren die Teilnehmenden im Durchschnitt 14.82 Jahre $(S D=0.50)$ alt, $56 \%$ von ihnen waren Mädchen.

\section{Instrumente}

Die Schüler*innen bewerteten ihre Berufswahlkompetenz mit Hilfe von 90 Items des Fragebogens zur Diagnostik von Berufswahlkompetenz von Kaak et al. (2013) (siehe Abschnitt 6.2). Ihre Antworten spiegeln ihre jeweilige Selbsteinschätzung auf den zwölf folgenden Facetten wider: Selbstwissen, Konzeptwissen, Bedingungswissen, Planungs- und Entscheidungswissen, Betroffenheit, Eigenverantwortung, Offenheit, Zuversicht, Exploration, Steuerung, Problemlösen und Stressmanagement.

\section{Statistisches Vorgehen}

Zur Identifikation der latenten Profile für die drei betrachteten Messzeitpunkte wurde die bereits in Studie 3 verwendete latente Profilanalyse (LPA) eingesetzt 
(vgl. Muthén \& Muthén, 1998-2017, siehe auch Abschnitt 8.2). Die darauf aufbauende latente Transitionsanalyse (LTA) eröffnet die Möglichkeit, Bewegungen der Schüler*innen zwischen den einzelnen Profilgruppen von Messzeitpunkt zu Messzeitpunkt sichtbar zu machen.

Wie in Abschnitt $8.2 \mathrm{im}$ Detail beschrieben ist, ermöglicht die LTA dabei nicht nur die Untersuchung heterogener Entwicklungsverläufe, sondern darüber hinaus auch die Betrachtung von unterschiedlich starken Veränderungen innerhalb verschiedener Zeitabschnitte. Aufgrund theoretischer Überlegungen wurden Kompetenzveränderungen über die Zeit angenommen, weshalb die Daten nicht auf Messinvarianz hin getestet wurden (vgl. auch Kia-Keating et al., 2018). Der Modellaufbau der LTA folgt dem von Nylund et al. (2008) beschriebenen schrittweisen Vorgehen. Um eine gegenseitige Beeinflussung der LPA-Modelle der einzelnen Messzeitpunkte zu verhindern, wurde zudem auf die neuere Drei-Stufen-Methode zurückgegriffen (Nylund-Gibson et al., 2014). Die latenten Analysen wurden in Mplus, Version 8 unter Verwendung der FIML-Methode berechnet (Graham, 2012). Alle weiteren Berechnungen wurden mit SPSS Statistics 25.0 durchgeführt.

Abschließend wurden die Schüler*innen anhand der identifizierten Bewegungsmuster eingeteilt in Mobile (Movers) und Verbleibende (Stayers) und ihre Bewegungsmuster entsprechend beschrieben. Abbildung 12.1 stellt das Pfaddiagramm der verwendeten LTA dar.

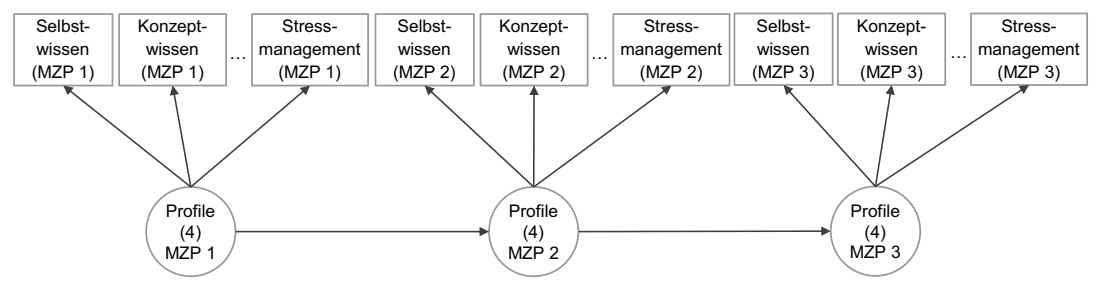

Abbildung 12.1 Darstellung der latenten Transitionsanalyse 


\subsection{Ergebnisse und Diskussion}

Die Resultate werden beginnend mit den querschnittlichen Modellen vorgestellt. Dafür werden zunächst die Fit-Indizes der LPA berichtet, die in Kombination mit weiteren theoretischen Überlegungen zur Entscheidung über die jeweilige Profilanzahl der drei Messzeitpunkte geführt haben. Anschließend folgt eine kurze Vorstellung der identifizierten Profile. Als nächstes werden die Profilgruppengrößen, die sich aus der LTA ergaben, für alle Messzeitpunkte präsentiert und die Bewegungsmuster des finalen LTA-Modells beschrieben und diskutiert.

Tabelle 12.1 stellt die Mittelwerte, Standardabweichungen und Cronbachs Alpha der zwölf Berufswahlkompetenzfacetten dar, die in den latenten Analysen jeweils als Indikatoren für die Profilgruppenbildung dienten.

Tabelle 12.1 Deskriptive Statistik der Berufswahlkompetenzfacetten für MZP 1 bis 3

\begin{tabular}{l|l|l|l|l|l|l|l|l|l}
\hline \multirow{2}{*}{ Skala (N Items) } & \multicolumn{3}{l}{ MZP 1 } & \multicolumn{3}{l}{ MZP 2 } & \multicolumn{3}{l}{ MZP 3 } \\
\cline { 2 - 13 } & $M$ & $S D$ & $\alpha$ & $M$ & $S D$ & $\alpha$ & $M$ & $S D$ & $\alpha$ \\
\hline Selbstwissen (9) & 3.11 & 0.49 & .80 & 3.11 & 0.52 & .84 & 3.18 & 0.49 & .83 \\
\hline Konzeptwissen (6) & 2.40 & 0.64 & .79 & 2.58 & 0.67 & .82 & 2.52 & 0.65 & .80 \\
\hline Bedingungswissen (7) & 2.74 & 0.73 & .85 & 2.78 & 0.71 & .86 & 2.79 & 0.73 & .88 \\
\hline $\begin{array}{l}\text { Planung \& Entscheidung } \\
\text { (4) }\end{array}$ & 2.60 & 0.70 & .68 & 2.64 & 0.73 & .71 & 2.57 & 0.75 & .67 \\
\hline Betroffenheit (8) & 3.51 & 0.41 & .77 & 3.35 & 0.59 & .87 & 3.43 & 0.48 & .78 \\
\hline Eigenverantwortung (6) & 3.02 & 0.57 & .79 & 3.01 & 0.61 & .82 & 2.95 & 0.62 & .80 \\
\hline Offenheit (7) & 2.54 & 0.53 & .69 & 2.52 & 0.60 & .78 & 2.41 & 0.58 & .75 \\
\hline Zuversicht (12) & 3.15 & 0.41 & .76 & 3.07 & 0.48 & .84 & 3.10 & 0.43 & .82 \\
\hline Exploration (9) & 2.00 & 0.60 & .85 & 2.04 & 0.66 & .89 & 1.89 & 0.67 & .89 \\
\hline Steuerung (7) & 2.98 & 0.51 & .71 & 2.94 & 0.58 & .80 & 2.90 & 0.59 & .79 \\
\hline Problemlösen (10) & 3.08 & 0.44 & .75 & 3.06 & 0.48 & .80 & 3.06 & 0.50 & .81 \\
\hline Stressmanagement (5) & 2.78 & 0.71 & .80 & 2.65 & 0.77 & .86 & 2.68 & 0.79 & .86 \\
\hline
\end{tabular}

\section{Latente Profilanalysen: Messmodelle und Berufswahlkompetenzprofile}

Um die Heterogenität des berufswahlbezogenen Entwicklungsstands der Jugendlichen zu untersuchen, wurde zunächst getrennt für die drei Messzeitpunkte eine Reihe von LPAs berechnet. Ausgangspunkt war ein latentes Modell mit nur einer Profilgruppe, das sukzessive um jeweils ein zusätzliches Profil hin bis zu insgesamt 
sechs Profilgruppen erweitert wurde. Die finale Anzahl der Profile wurde für die einzelnen Messzeitpunkte jeweils separat bestimmt. Da der Modellfit nicht an einem einzelnen Entscheidungsfaktor bestimmt werden kann, wurden mehrere Indikatoren in der Modellbewertung berücksichtigt, nämlich BIC, SaBIC, BLRT, VLMRT sowie der Log-Likelihood-Wert und der Entropiewert. Aus Gründen der Interpretierbarkeit wurden bei der Entscheidungsfindung darüber hinaus auch theoretische Aspekte sowie Klassengrößen und durchschnittliche latente Klassenwahrscheinlichkeiten berücksichtigt.

Tabelle 12.2 gibt einen Überblick über die Fit-Indizes. Allein anhand der aufgeführten Fit-Indizes zeichnete sich kein Modell als offensichtlich bestgeeignetes aus. Für alle Messzeitpunkte indizieren BIC, SaBIC sowie die Log-LikelihoodWerte einen besseren Fit für Modelle mit einer höheren Profilanzahl (vier oder mehr Profile). Während dessen weisen die Signifikanzen des VLRMT auf eine ZweiProfile-Lösung für den ersten Messzeitpunkt, eine Zwei- oder Drei-Profile-Lösung für den zweiten Messzeitpunkt und ein Zwei- oder Vier-Profile-Lösung für den dritten Messzeitpunkt hin. Da die Werte des BLRT für alle Modelle signifikant waren, können daraus keine zusätzlichen Schlussfolgerungen gezogen werden. Auch die Entropiewerte deuteten auf keine bestimmte Profilanzahl hin. In Abwägung theoretischer Faktoren sowie der Klassengrößen schien eine Vier-Profile-Lösung für alle drei Messzeitpunkte am sinnvollsten.

Abbildung 12.2 stellt die Ausgangslage der Schüler*innen hinsichtlich der zwölf Berufswahlkompetenzfacetten zum ersten Messzeitpunkt dar. Es lassen sich drei Profile mit einem ähnlichen Muster erkennen, die sich hauptsächlich im allgemeinen Entwicklungsstand unterscheiden, ansonsten aber ähnliche Muster aufweisen. Das vierte Profil zeichnet sich jedoch durch ein stark abweichendes Muster der Berufswahlkompetenz aus. Diese Befunde decken sich mit denen der vorangegangenen Studie (siehe Kapitel 11), die anhand des dritten Messzeitpunkts der gleichen Stichprobe durchgeführt wurde. Dort wurden die Lernenden der ersten drei Profilgruppen aufgrund der Niveauunterschiede in der Berufswahlkompetenzausprägung als Unkonkrete mit einfach ausgeprägtem Kompetenzprofil, Konkretisierende mit durchschnittlich ausgeprägtem Kompetenzprofil und Fortgeschrittene mit stark ausgeprägtem Kompetenzprofil bezeichnet.

Die Schüler*innen der vierten Profilgruppe erscheinen ihrerseits irgendwie sorgenfrei. Sie schätzen ihre Fähigkeiten zur Problemlösung und Stressbewältigung vergleichsweise hoch ein. Gleichzeitig scheinen sie relativ wenig über die Arbeitswelt und ihre Voraussetzungen zu wissen, wenig zu planen und wenig Offenheit für berufliche Alternativen zu zeigen oder diese zu erkunden. Sie scheinen zuversichtlich, dass sich Optionen und Pläne eventuell ergeben werden und sie ihre 
Tabelle 12.2 LPA Modellfit-Indizes für MZP 1 bis 3

\begin{tabular}{|c|c|c|c|c|c|c|}
\hline$N$ Profile & Log-likelihood & Entropie & BIC & SaBIC & $\begin{array}{l}\text { BLRT } \\
p \text {-Wert }\end{array}$ & $\begin{array}{l}\text { VLRMT } \\
p \text {-Wert }\end{array}$ \\
\hline \multicolumn{7}{|c|}{ LPA MZP 1} \\
\hline 1 & -2870.11 & 1.00 & 5877.10 & 5800.99 & $\mathrm{n} / \mathrm{a}^{\mathrm{a}}$ & $\mathrm{n} / \mathrm{a}^{\mathrm{a}}$ \\
\hline 2 & -2505.77 & 0.85 & 5222.58 & 5105.24 & 0.0001 & 0.00 \\
\hline 3 & -2403.56 & 0.83 & 5092.31 & 4933.74 & 0.0001 & 0.21 \\
\hline 4 & -2342.23 & 0.83 & 5043.81 & 4844.01 & 0.0001 & 0.11 \\
\hline 5 & -2286.62 & 0.85 & 5006.73 & 4765.70 & 0.0001 & 0.39 \\
\hline 6 & -2243.96 & 0.86 & 4995.56 & 4713.31 & 0.0001 & 0.71 \\
\hline \multicolumn{7}{|c|}{ LPA MZP 2} \\
\hline 1 & -3613.74 & 1.00 & 7366.79 & 7290.66 & $\mathrm{n} / \mathrm{a}^{\mathrm{a}}$ & $\mathrm{n} / \mathrm{a}^{\mathrm{a}}$ \\
\hline 2 & -3171.40 & 0.88 & 6557.58 & 6440.21 & 0.0001 & 0.00 \\
\hline 3 & -3008.25 & 0.87 & 6306.57 & 6148.16 & 0.0001 & 0.00 \\
\hline 4 & -2956.28 & 0.85 & 6278.28 & 6078.44 & 0.0001 & 0.67 \\
\hline 5 & -2897.76 & 0.84 & 6236.70 & 5995.63 & 0.0001 & 0.24 \\
\hline 6 & -2852.20 & 0.86 & 6221.06 & 5938.75 & 0.0001 & 0.50 \\
\hline \multicolumn{7}{|c|}{ LPA MZP 3} \\
\hline 1 & -3232.44 & 1.00 & 6601.86 & 6525.74 & $\mathrm{n} / \mathrm{a}^{\mathrm{a}}$ & $\mathrm{n} / \mathrm{a}^{\mathrm{a}}$ \\
\hline 2 & -2830.51 & 0.88 & 5872.19 & 5754.85 & 0.0001 & 0.00 \\
\hline 3 & -2722.88 & 0.87 & 5731.12 & 5572.55 & 0.0001 & 0.17 \\
\hline 4 & -2635.99 & 0.89 & 5631.52 & 5431.72 & 0.0001 & 0.01 \\
\hline 5 & -2587.97 & 0.89 & 5609.69 & 5368.66 & 0.0001 & 0.09 \\
\hline 6 & -2553.91 & 0.88 & 5615.76 & 5333.50 & 0.0001 & 0.14 \\
\hline
\end{tabular}

Anmerkung. $\mathrm{BIC}=$ Bayesian Information Criteria, $\mathrm{SaBIC}=$ Adjusted $\mathrm{BIC}, \mathrm{BLRT}=$ Bootstrapped Likelihood Ratio Test, VLRMT = Vuong-Lo-Mendell-Rubin Likelihood Ratio Test $* p<.05, * * p<.01, * * * p<.001$

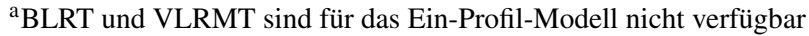

beruflichen Ziele mit ihren bestehenden Fähigkeiten erreichen können. Die Schüler*innen der vierten Profilgruppe wurde als die Fokussierten mit stark variierenden Kompetenzausprägungen bezeichnet. Diese Terminologie wird hier entsprechend übernommen.

Tabelle 12.3 gibt Auskunft über die prozentuale Verteilung der Schüler*innen auf die vier Profilgruppen zu allen drei Messzeitpunkten. Die Konkretisierenden 


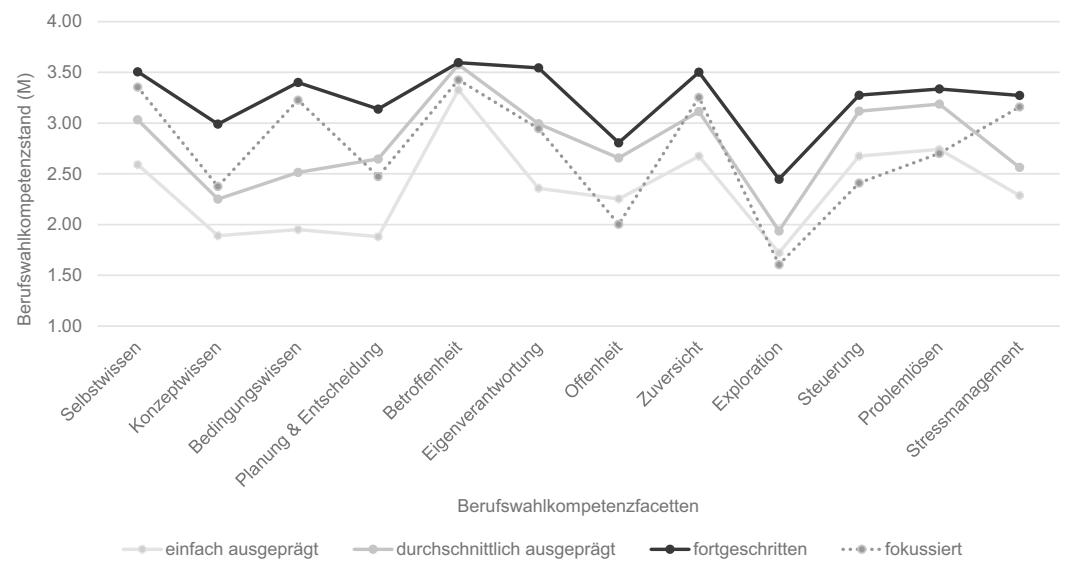

Abbildung 12.2 Mittelwerte der Berufswahlkompetenzfacetten der vier Profile (MZP 1)

stellen mit bis zu $47 \%$ (MZP 2) in allen drei Umfragen die größte Gruppe, gefolgt von den Fortgeschrittenen (21 - $35 \%$ ). Je nach Messzeitpunkt weisen $13 \%$ bis $22 \%$ der befragten Schüler*innen ein einfach ausgeprägtes Kompetenzprofil auf. Die Fokussierten repräsentieren mit $10 \%$ bis $13 \%$ der Befragten ebenfalls eine kleine, aber nicht zu vernachlässigende Gruppe.

Tabelle 12.3 Prozentuale Verteilung der

Schüler*innen auf die Berufswahlkompetenzprofile für MZP 1 bis 3 (LPA)

\begin{tabular}{l|l|l|l}
\hline Profil & MPZ 1 & MPZ 2 & MPZ 3 \\
\hline Die Unkonkreten (U) & $22 \%$ & $20 \%$ & $13 \%$ \\
\hline Die Konkretisierenden (K) & $36 \%$ & $47 \%$ & $41 \%$ \\
\hline Die Fortgeschrittenen (F) & $30 \%$ & $21 \%$ & $35 \%$ \\
\hline Die Fokussierten (FO) & $13 \%$ & $13 \%$ & $10 \%$ \\
\hline
\end{tabular}

\section{Befunde der latenten Transitionsanalyse: Bewegungsmuster}

Nach der Entscheidung über die Anzahl der Profile für die einzelnen Messzeitpunkte galt es zu prüfen, ob und wie sich die Schüler*innen im Laufe der Zeit zwischen den Profilgruppen bewegen. Zur Klärung dieser Frage wurden die Profile, die den einzelnen Schüler*innen für jeden Messpunkt zugeordnet wurden, als Indikatoren in die LTA eingeführt. Die daraus resultierende Verteilung der Schüler*innen auf 
die vier Profilgruppen ist in Tabelle 12.4 für jeden Messzeitpunkt dargestellt. Die Konkretisierenden stellen weiterhin die größte Gruppe. Der prozentuale Anteil der Schüler*innen, die zur Gruppe der Fokussierten gehören, ging je nach Messzeitpunkt auf $4 \%$ bis $9 \%$ zurück. Mit Ausnahme des dritten Messzeitpunkts, zu dem sie $31 \%$ der Stichprobe ausmachen, blieb der Anteil derjenigen mit einem einfach ausgeprägten Berufswahlkompetenzprofil relativ stabil.

Tabelle 12.4 Prozentuale

Verteilung der

Schüler*innen auf die

Berufswahlkompetenzpro-

file für MZP 1 bis 3

(LTA)

\begin{tabular}{l|l|l|l}
\hline Profil & MZP 1 & MZP 2 & MZP 3 \\
\hline Die Unkonkreten (U) & $22 \%$ & $31 \%$ & $16 \%$ \\
\hline Die Konkretisierenden (K) & $41 \%$ & $43 \%$ & $49 \%$ \\
\hline Die Fortgeschrittenen (F) & $27 \%$ & $22 \%$ & $28 \%$ \\
\hline Die Fokussierten (FO) & $9 \%$ & $4 \%$ & $6 \%$ \\
\hline
\end{tabular}

Bei der Analyse der verschiedenen Bewegungsmuster, die sich über die drei Messzeitpunkte ergaben, kristallisierten sich insgesamt 27 Entwicklungsmuster heraus, die die erste Hypothese unterschiedlicher Verlaufsbahnen stützen (siehe Tabelle 12.5).

Grundsätzlich konnten zwei übergreifende Bewegungsarten identifiziert werden, nämlich die Verbleibenden (stayers) und die Mobilen (movers). Als Verbleibende wurden diejenigen Schüler*innen betitelt, die dauerhaft in ihrer ursprünglichen Profilgruppe verweilen. Sie umfassen $43 \%$ der Gesamtstichprobe. Bemerkenswert ist, dass keine einzige Person dauerhaft zu den Fokussierten zählte. Allerdings schätzen $10 \%$ der Schüler*innen ihre Berufswahlkompetenz über den gesamten Betrachtungszeitraum hinweg als einfach entwickelt ein. Diese Gruppe formt aufgrund der fehlenden Entwicklung ihrer Berufswahlkompetenz eindeutig eine Risikogruppe. Angesichts der Verbleibenden muss die zweite Hypothese hinsichtlich der Profilbewegungen teilweise verworfen werden.

Die zweite Gruppe der Bewegungsmuster, die Mobilen, umfassen $57 \%$ der befragten Schüler*innen. Innerhalb der Gruppe der Mobilen lassen sich vier Untergruppen differenzieren: Die erste Untergruppe (22\%, upward movers) vollzieht in ihrer Berufswahlkompetenzeinschätzung insgesamt eine Aufwärtsbewegung in eine Profilgruppe mit höherem Berufswahlkompetenzniveau. Folglich kann davon ausgegangen werden, dass diese Jugendlichen einen Berufswahlkompetenzerwerb erfahren. $1 \%$ der Gesamtstichprobe bzw. $3 \%$ der Jugendlichen, die zum ersten Messzeitpunkt zu den Unkonkreten mit einem einfach ausgeprägten Kompetenzprofil gehörten, verbesserten sich hin zur Gruppe der Fortgeschrittenen. 
Tabelle 12.5 Prozentualer Anteil der Schüler*innen in jedem Bewegungsmuster, geordnet nach Bewegungsrichtung

\begin{tabular}{|c|c|c|c|c|}
\hline & MZP 1 & MZP 2 & MZP 3 & Häufigkeit \\
\hline \multicolumn{5}{|l|}{ Mobile (57 \%) } \\
\hline \multirow{8}{*}{ Upward Movers } & $\mathrm{K}$ & $\mathrm{F}$ & $F$ & $1 \%$ \\
\hline & $\mathrm{K}$ & FO & F & $1 \%$ \\
\hline & $\mathrm{K}$ & $\mathrm{K}$ & $\mathrm{F}$ & $8 \%$ \\
\hline & $\mathrm{U}$ & $\mathrm{U}$ & F & $1 \%$ \\
\hline & $\mathrm{U}$ & F & $\mathrm{K}$ & $1 \%$ \\
\hline & $\mathrm{U}$ & K & $\mathrm{K}$ & $3 \%$ \\
\hline & $\mathrm{U}$ & $\mathrm{U}$ & $\mathrm{K}$ & $6 \%$ \\
\hline & FO & $\mathrm{F}$ & $\mathrm{F}$ & $1 \%$ \\
\hline \multirow[t]{5}{*}{ Returning Movers } & FO & $\mathrm{U}$ & FO & $3 \%$ \\
\hline & $\mathrm{K}$ & $\mathrm{F}$ & $\mathrm{K}$ & $2 \%$ \\
\hline & $\mathrm{K}$ & $\mathrm{U}$ & $\mathrm{K}$ & $3 \%$ \\
\hline & $\mathrm{K}$ & FO & $\mathrm{K}$ & $2 \%$ \\
\hline & $\mathrm{F}$ & $\mathrm{K}$ & $\mathrm{F}$ & $5 \%$ \\
\hline \multirow[t]{5}{*}{ Diffuse Movers } & $\mathrm{F}$ & $\mathrm{F}$ & FO & $1 \%$ \\
\hline & $\mathrm{K}$ & $\mathrm{U}$ & FO & $1 \%$ \\
\hline & FO & $\mathrm{F}$ & $\mathrm{K}$ & $1 \%$ \\
\hline & FO & $\mathrm{U}$ & $\mathrm{K}$ & $1 \%$ \\
\hline & $\mathrm{U}$ & $\mathrm{U}$ & FO & $2 \%$ \\
\hline \multirow[t]{3}{*}{ Downward Movers } & $\mathrm{F}$ & $\mathrm{F}$ & $\mathrm{K}$ & $4 \%$ \\
\hline & $\mathrm{F}$ & FO & $\mathrm{K}$ & $1 \%$ \\
\hline & $\mathrm{F}$ & K & K & $5 \%$ \\
\hline Risikogruppe & FO & $\mathrm{U}$ & $\mathrm{U}$ & $3 \%$ \\
\hline Risikogruppe & $\mathrm{K}$ & $\mathrm{U}$ & $\mathrm{U}$ & $2 \%$ \\
\hline Risikogruppe & $\mathrm{K}$ & $\mathrm{K}$ & $\mathrm{U}$ & $1 \%$ \\
\hline \multirow[t]{2}{*}{ Verbleibende (43\%) } & $\mathrm{F}$ & $\mathrm{F}$ & $\mathrm{F}$ & $11 \%$ \\
\hline & $\mathrm{K}$ & $\mathrm{K}$ & $\mathrm{K}$ & $21 \%$ \\
\hline Risikogruppe & $\mathrm{U}$ & $\mathrm{U}$ & $\mathrm{U}$ & $10 \%$ \\
\hline
\end{tabular}

Anmerkung. $\mathrm{U}=$ Unkonkrete, $\mathrm{K}=$ Konkretisierende, $\mathrm{F}=$ Fortgeschrittene, $\mathrm{FO}=$ Fokussierte; Die Musterhäufigkeit ist prozentual zur Gesamtstichprobe angegeben. Für die Beschreibung der Bewegungsmuster werden die englischen Originalnamen verwendet. 
Die zweite Untergruppe der Mobilen (15\%, returning movers) bewegt sich zwar im Laufe der Zeit zwischen verschiedenen Profilen hin und weg, kehrt aber letztlich zu ihrem ursprünglichen Kompetenzprofil zurück. Hier sind auch diejenigen Schüler*innen mit einbezogen, die am Ende erneut zu den Fokussierten gehören ( $3 \%$ ). Überraschenderweise kehrten Schüler*innen, sobald sie einen einfachen Berufswahlkompetenzstand überwunden hatten, nicht mehr auf dieses Niveau zurück.

Daraus kann die Hypothese abgeleitet werden, dass Schüler*innen mit einem anfangs einfach ausgeprägten Berufswahlkompetenzprofil dahin nicht wieder zurückfallen, sobald ihre Entwicklung in Bewegung gekommen ist und einen bestimmten Punkt erreicht hat. Mit Blick auf den Praxistransfer gilt es künftig genauer zu untersuchen, inwieweit frühzeitig wahrgenommene Angebote zur beruflichen Orientierung für Schüler*innen mit einem anfänglich niedrigen Berufswahlkompetenzstand Langzeiteffekte entwickeln könnten.

Die dritte Untergruppe der Mobilen (downward movers), die $16 \%$ der Gesamtstichprobe abdeckt, findet sich schließlich in einer Profilgruppe mit einem niedrigeren berufswahlbezogenen Gesamtniveau wieder. $6 \%$ aller Teilnehmenden befanden sich aufgrund ihrer Selbsteinschätzungen letztlich sogar in der Gruppe der Unkonkreten wieder. Sie schätzten folglich ihre Berufswahlkompetenz als gering ausgeprägt ein und bilden damit ebenfalls eine potentielle Risikogruppe. Aufgrund dieser Gruppe muss die dritte Hypothese, dass alle Schüler*innen im Laufe der Zeit eine positive Entwicklung ihrer Berufswahlkompetenz erfahren, abgelehnt werden.

Erwähnenswert ist in dieser Stichprobe jedoch auch, dass Schüler*innen, die ihre Berufswahlkompetenz initial als fortgeschritten einschätzten, zu keinem Zeitpunkt auf ein einfach ausgeprägtes Kompetenzprofil absanken.

In der vierten Untergruppe der Mobilen (4\%, diffuse movers) befinden sich die Schüler*innen, deren Berufswahlkompetenzeinschätzungen nicht klassifizierbaren, diffusen Bewegungen folgten. Sie beginnen oder enden entweder als Fokussierte, einige von ihnen zeigten schließlich ein durchschnittlich ausgeprägtes Berufswahlkompetenzprofil. Da die detaillierte Analyse des Profils der Fokussierten noch aussteht, lässt sich nur schwer beurteilen, ob eine Bewegung hinzu diesem Profil oder weg von ihm in Richtung eines durchschnittlich ausgeprägten Kompetenzstands als positive oder negative Entwicklung angesehen werden muss. 


\subsection{Limitationen und Schlussfolgerungen}

Zusammenfassend lässt sich sagen, dass die vorliegende Studie nur einen ersten Versuch darstellt, latente Bewegungsmuster von Schüler*innen in ihrem Berufswahlkompetenzerleben bzw. ihrer Entwicklung zu erschließen.

Der verwendete personenzentrierte und modellbasierte Ansatz bietet sich für die Untersuchung des Berufswahlkompetenzkonstrukts an, da er erlaubt, homogene Profilgruppen zu betrachten, von denen ausgehend zudem unterschiedliche Bewegungsverläufe distinguiert werden können.

Die Resultate dieser Studie zeigen vier verschiedene Profilgruppen, in die Schüler*innen entsprechend eines einfach, durchschnittlich oder stark ausgeprägten Kompetenzprofils bzw. eines Profils mit stark variierenden Kompetenzausprägungen eingeteilt werden können. Im Einklang mit früheren Forschungsergebnissen (vgl. Kapitel 11) unterscheiden sich drei Profile hauptsächlich über ihr Gesamtniveau der Berufswahlkompetenz, während das vierte Profil auch im Kompetenzmuster abweicht.

Darüber hinaus zeigte sich, dass $43 \%$ der Schüler*innen ihr Kompetenzniveau über den beobachteten Zeitraum konstant halten, während die anderen $57 \% \mathrm{im}$ Laufe der Zeit zwischen den Profilen wechseln. Es wurde auch deutlich, dass rund $16 \%$ der Stichprobe eine potentielle Risikogruppe konstituieren. Ihre Berufswahlkompetenz wies zum letzten Messpunkt nur eine geringe Konkretisierung auf bzw. wurde von den Jugendlichen als einfach ausgeprägt eingeschätzt.

Angesichts eines großen Anteils von Verbleibenden, also Schüler*innen, die ihre Berufswahlkompetenz konstant gleich einschätzen, stellt sich die Frage, inwieweit Berufswahlkompetenz und ihre Facetten überhaupt als flexibel angesehen werden können oder ob Facetten wie berufswahlbezogene Offenheit, Explorationsverhalten oder berufswahlbezogenes Stressmanagement vielmehr stabile Eigenschaften darstellen.

Befunde früherer Studien, die nur geringe oder keinerlei Effekte von berufsorientierenden Maßnahmen auf die berufliche Orientierung festgestellt haben (Ratschinski \& Struck, 2016), könnten diese Argumentation stützen. Allerdings wurden die Effekte nicht auf bestehende Untergruppen hin geprüft.

Zukünftige Forschung müsste folglich untersuchen, ob der Erwerb von Berufswahlkompetenz gefördert werden kann, indem berufsorientierende Maßnahmen an jeweils spezifische Bedürfnisse der Profilgruppen angepasst werden. Zudem bleiben mögliche Unterschiede, aber auch Gemeinsamkeiten zwischen der angeführten Risikogruppe und denjenigen Schüler*innen, die einen starken Kompetenzzuwachs erleben konnten, zu erforschen. Unterschiede könnten beispielsweise Auswirkungen von besuchten berufsorientierenden Maßnahmen sein oder auf 
soziodemographische Unterschiede (Ohlemann \& Ittel, 2017) bzw. die wahrgenommene soziale Unterstützung (Garcia, Restubog, Bordia, Bordia \& Roxas, 2015) zurückführbar sein.

Die Ergebnisse müssen jedoch vor dem Hintergrund der relativ kleinen Stichprobe und dem Fehlen von Kovariaten in ihrer Interpretation eingeschränkt werden.

Daher sollen in einem neuen Längsschnittprojekt mit einer größeren und diverseren Stichprobe perspektivisch auch mögliche Schul- und Klasseneffekte untersucht werden. In diesem Projekt werden auch weitere Kovariaten wie das Alter und die Muttersprache der Schüler*innen, ihre Schulleistungen sowie Einflüsse des sozialen Umfelds und berufsorientierende Aktivitäten in der Schule in die Analysen integriert werden. In der wissenschaftlichen Debatte, aber auch mit und zwischen Schulen und Maßnahmenanbietenden könnte das Wissen um die unterschiedlichen Bewegungsmuster die Wahrnehmung individualisierter Unterstützung beruflicher Orientierungsprozesse als wirksame Maßnahme von Inklusion und Chancengleichheit schärfen.

Open Access Dieses Kapitel wird unter der Creative Commons Namensnennung 4.0 International Lizenz (http://creativecommons.org/licenses/by/4.0/deed.de) veröffentlicht, welche die Nutzung, Vervielfältigung, Bearbeitung, Verbreitung und Wiedergabe in jeglichem Medium und Format erlaubt, sofern Sie den/die ursprünglichen Autor(en) und die Quelle ordnungsgemäß nennen, einen Link zur Creative Commons Lizenz beifügen und angeben, ob Änderungen vorgenommen wurden.

Die in diesem Kapitel enthaltenen Bilder und sonstiges Drittmaterial unterliegen ebenfalls der genannten Creative Commons Lizenz, sofern sich aus der Abbildungslegende nichts anderes ergibt. Sofern das betreffende Material nicht unter der genannten Creative Commons Lizenz steht und die betreffende Handlung nicht nach gesetzlichen Vorschriften erlaubt ist, ist für die oben aufgeführten Weiterverwendungen des Materials die Einwilligung des jeweiligen Rechteinhabers einzuholen. 